\title{
Risks of space colonization
}

\author{
Marko Kovic*
}

July 2020

\begin{abstract}
Space colonization is humankind's best bet for long-term survival. This makes the expected moral value of space colonization immense. However, colonizing space also creates risks - risks whose potential harm could easily overshadow all the benefits of humankind's longterm future. In this article, I present a preliminary overview of some major risks of space colonization: Prioritization risks, aberration risks, and conflict risks. Each of these risk types contains risks that can create enormous disvalue; in some cases orders of magnitude more disvalue than all the potential positive value humankind could have. From a (weakly) negative, suffering-focused utilitarian view, we therefore have the obligation to mitigate space colonization-related risks and make space colonization as safe as possible. In order to do so, we need to start working on real-world space colonization governance. Given the near total lack of progress in the domain of space governance in recent decades, however, it is uncertain whether meaningful space colonization governance can be established in the near future, and before it is too late.
\end{abstract}

*markokovic@gmail.com 


\section{Introduction: The value of colonizing space}

Space colonization, the establishment of permanent human habitats beyond Earth, has been the object of both popular speculation and scientific inquiry for decades. The idea of space colonization has an almost poetic quality: Space is the next great frontier, the next great leap for humankind, that we hope to eventually conquer through our force of will and our ingenuity. From a more prosaic point of view, space colonization is important because it represents a long-term survival strategy for humankind 1 .

Space colonization is tremendously important for the future of humankind in two ways. First, space colonization means that the total future number of humans who will exist and whose lives will be worth living could be orders of magnitude greater than today [2, 3, 4]. By colonizing space, humankind could therefore create a future that is generally morally desirable: There could be vastly more people to enjoy vastly more life-years worth living if we succeed in colonizing space. Second, engaging in space colonization represents a strategy for mitigating existential risks. Existential risks are risks that could result in the extinction of humankind or in the permanent curtailing of humankind's potential for future development [5]. In a more technical sense, existential risks can be thought of as risks that could cause the permanent loss of a large fraction of humankind's future moral expected value [6]: If humankind goes extinct or stagnates prematurely, the majority of humankind's positive future value (the many thousands of generations and many billions of people who could lead lives worth living) would be lost. Mitigating existential risks is therefore a moral priority, even though the current generation of humans and other sentient beings might not be affected by them in their own lifetimes.

We do not know when, or if at all, humankind will achieve the technological capability for creating permanent habitats beyond Earth. But if space colonization were merely uncertain in this manner, humankind should still push for space colonization as much and as fast as possible, since the added value of achieving space colonization capabilities sooner rather than later would be big, and the added value of increasing the overall probability of achieving space colonization capabilities at all would be immense. But space colonization is not just uncertain. It is also risky. Engaging in space colonization and eventually colonizing space can exacerbate existing risks and it can create new ones. The risks so exacerbated and created could create a

\footnotetext{
${ }^{1}$ For the purpose of this article, I use "long-term" to denote a time frame that is at least somewhat cognitively palpable and practically tractable: Thousands of years. The long long-term future of humankind, however, could easily play out on a time scale of hundreds of thousands of years if we take the average mammalian species lifetime of around one millions years [1] as a baseline.
} 
lot of moral disvalue by removing happiness and by creating suffering - in some scenarios so much so that the overall benefit of colonizing space could end up being smaller than the damage caused by it.

\subsection{The purpose of this article}

The goal of this article is to bring greater academic attention to the, in my view, neglected question of space colonization-related risks. In order to do so, I present a preliminary conceptual survey of relevant risks of space colonization.

The scope of the present article is limited in two important ways. First, the risk types and specific risks within those types that I discuss in sections 2 to 5 are almost certainly not exhaustive. My ambition with this article is not for it to be a final authoritative voice on the issue of space colonization-related risks, but rather a first attempt at increasing conceptual clarity. Second, even though I briefly address the challenge of space colonization governance in section 6, my primary aim with this article is point out the existence of a class of problems, not to already offer solutions for them. Future research should engage in more detailed discussions on how to mitigate space colonizationrelated risks, but the purpose of article is to start a discussion on what those risks are. Metaphorically speaking, the present article is an attempt at mapping out the terrain in a first draft; as the map becomes more accurate and precise in future iterations thanks to more research, the best path for avoiding the major pitfalls will become clearer as well.

\section{Conceptualizing space colonization-related risks}

Pursuing and engaging in space colonization activities creates a myriad of risks. For example, there would almost certainly be fatal accidents similar to the Challenger disaster of 1986 and the Columbia disaster of 2003; there would almost certainly be smaller workplace-related accidents like broken legs and arms; there would almost certainly be workplace-related issues such as sexual harassment or mobbing or burnout; there would almost certainly be people who are deeply saddened or offended by the mere idea of colonizing space; and so forth. All of these risks are real and important, but compared to the immense benefit of colonizing space, they seem relatively minor. Of course, every instance of sexual harassmant or mobbing and every unnecessary death is one that should ideally be avoided, but those types of risks do not really put into question the overall utilitarian big picture: Undesirable 
though such minor or limited risks are, the positive value that can be created by colonizing space is much greater. But that is not necessarily universally true for all risks that could be created due to space colonization. Colonizing space also creates risks that whose amount of potential disvalue is big enought to put into question the overall value of colonizing space.

As a starting point for describing this category of more relevant space colonization-related risks, I use the risk assessment framework developed by Bostrom [5]. Bostrom's framework is depicted in Figure 1.

Figure 1: Risk analysis framework developed by Bostrom [5].

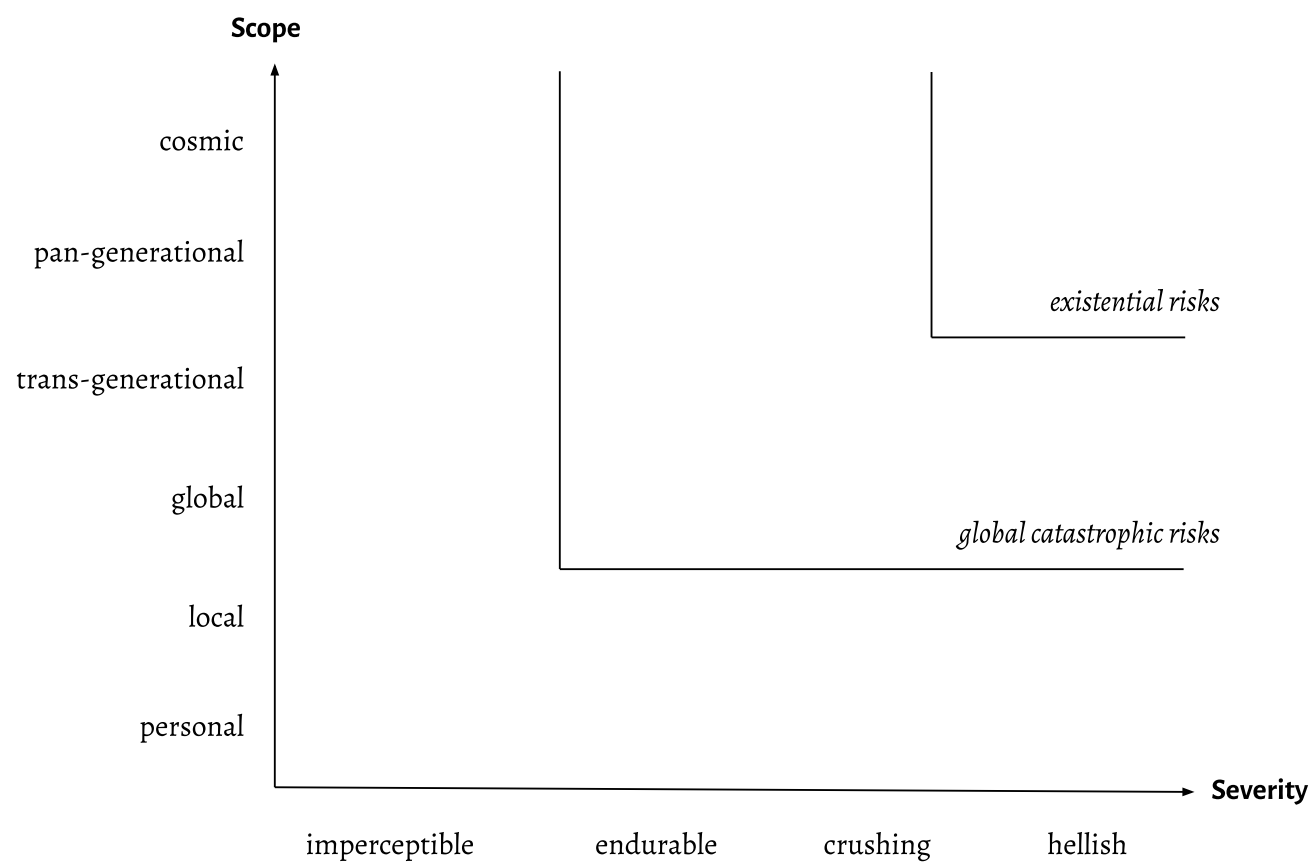

The focus of Bostrom's work are risks that are at least global in scope (in the sense of affecting all humans and potentially other sentient beings) and that have a significant negative impact. Within this space of what can be called grave risks, there are two important risk categories: Global catastrophic risks and existential risks. Global catastrophic risks are risks that create enormous moral disvalue in the form of suffering, but that do not, for example, threaten humankind's continued existence. Global catatstrophic risks are immensely bad, but not terminally so. Existential risks, on the other hand, are risks that directly threaten humankind's existence, be it in the form of extinction or in the form of permanent and premature curtailing of humankinds potential for future development. 
These two categories of grave risks, global catastrophic risks and existential risks, form the first dimension I use for conceptualizing space colonizationrelated risks. We should be interested in those two categories of space colonization-related risks because they are grave enough to either significantly reduce the overall benefit of colonizing space or to even erase it altogether and create more disvalue than value. The second dimension I use for conceptualizing space colonization-related risks is the distinction between, on the one hand, risks that already exist and that are being exacerbated by engaging in space colonization and, on the other hand, entirely new risks that would not otherwise exist if it weren't for humankind's space colonization activities. We can call this dimension the causal function of space colonization. When these two dimensions are combined, we arrive at a matrix depicted in Figure 2,

Figure 2: Framework for conceptualizing space colonization-related risks.

\begin{tabular}{|c|c|c|c|}
\hline & \multicolumn{2}{|c|}{ Causal function of space colonization } \\
\hline & & $\begin{array}{l}\text { exacerbating } \\
\text { existing risks }\end{array}$ & $\begin{array}{l}\text { creating } \\
\text { new risks }\end{array}$ \\
\hline & catastrophic & prioritization risks & conflict risks \\
\hline & existential & prioritization risks & $\begin{array}{c}\text { aberration risks } \\
\text { conflict risks }\end{array}$ \\
\hline
\end{tabular}

The cells in Figure 2 are populated with three types or classes of risks: Prioritization risks, aberration risks and conflict risks. These risk types are the ones I propose and focus on in this article, but they do not necessarily represent an exhaustive typology of risks within the proposed framework.

Prioritization risks are risks that arise either because addressing existing risks is potentially a more effective way of arriving at a morally desirable long-term future, or because pursuing space colonization could speed up the rate at which risks are created.

Aberration risks are risks that might cause great harm in the form of inadvertent collateral damage. These risks are entirely new risks for which there is no prior experience or knowledge or precedent from human history. They are a risk type sui generis for two main reasons: They expand the moral circle beyond humankind, and the disvalue they create is potentially orders of magnitude greater than the positive value of a future in which humankind has successfully colonized space 
Conflict risks are risks of violent conflict in the context of space colonization activities. The scale of these potential conflicts is much greater than anything experienced in human history so far, and the amount of disvalue created by them is can be immense.

In the sections that follow, I delve into these three risk types and describe more specific risks and risk scenarios within each risk type. Before doing so, however, it is necessary to briefly address the usefulness of the concept of existential risks. Much of the argument in this article rests on the assumption that there is such a thing as existential risk. If that is not the case, most of the space colonization-related risks I discuss disappear.

\subsection{Are existential risks real?}

Assessing risks is usually contingent on some prior experiential, empirical foundation. We can calculate the risks of driving a car for 100 kilometres because there is plenty of data on road accidents. We can calculate the risk of dying from an infectious disease like the seasonal flu because there is plenty of data on health outcomes following an infection with the seasonal influenza virus. We can even calculate or estimate some catastrophic risks because humankind has experienced them before (regional or global pandemics, world wars, aging and aging-related suffering, and so forth). But humankind has, by definition, never experienced an existential risk. We might intellectually understand that humankind might some day not exist anymore the way it exists today, but the probability of that state of the world becoming a reality any time soon is miniscule. After all, humankind has so far managed to survive all catastrophic risks thrown at it, and cataclysmic events such as a large comet or asteroid impact are extremely rare.

In light of this somewhat untangible nature of existential risks, some scholars and public commentator ${ }^{2}{ }^{2}$ reject the notion of existential risks altogether. The major criticism levelled against the concept of existential risks is that it represents something akin to a modern Pascal's wager [8]: No matter how improbable existential risks are, one can always make the better-safethan-sorry argument in favor of addressing them, since even the sligthest probability or even the mere possibility of the existence of an existential risk means that the existential risk in question warrants attention. So just as it is the safe option to believe in god because the prospect of an eternity in hell is terrible, it is the safe option to believe in existential risks because the prospect of an existential risk causing harm is terrible.

There are at least three counterarguments to this criticism. First, when

\footnotetext{
${ }^{2}$ Perhaps most notably the well-known public intellectual Steven Pinker [7, 290-321].
} 
thinking about existential risk, we are prone to underestimating risk probabilities because the probabilities in question are typically much smaller than the probability of that estimate being flawed [9], and we are likely to fall prey to a variation of the anthropic bias $[10]^{3}$

Second, the seeming improbability of existential risks usually has to do with natural existential risks. Natural existential risks are indeed very rare, and they are iid (independent and identically distributed) risks. Risks such as a devastating Solar flare or a devastating comet impact or a devastating mega quake are iid: They are rare, and their probability is not affected by prior outcomes or by human activity. But there is a crucial second class of existential risks: Anthropogenic, man-made existential risks. Anthropogenic existential risks are not iid risks. They are a direct consequence of human technological development, and they are increasing in probability and number as technological progress continues [14]. For Uncontrollable artificial superintelligence [15, 16], climate change-induced extreme heat stress [17] or ecosystem collapse [18, and the accidental or targeted misuse of biotechnology [19] are risks that have only recently emerged due to humankind's technological progress, and they are already more probable than natural existential risk 4

Third, the concept of existential risks is actually quite tangible if we extend our analytical gaze to non-human species. The extinction of species is a well-studied field, and homo sapiens sapiens is ultimately also simply a species that came about through the same evolutionary process as all other species have. If we accept our fundamental similarity to other life forms and species on Earth, tere is nothing inherently implausible about the notion that humankind might be on the verge of non-existence of permanent stagnation.

\footnotetext{
${ }^{3}$ Anthropic biases are observer effects which arise when our empirical observations are affected or biased by the necessity of us, the observers, existing. A simple anthropic bias in the context of existential risk is the fact that many potential existential risks mean that we, the observers, will be gone if the existential risks were to manifest. This amounts to a kind of survivorship bias [1]: The mere fact that we exist right now might intuitively suggest that there is not much to existential risks. But that inference is incorrect. Indeed, the opposite might be true, as proposed in the doomsday argument [12, [13, 89-108].

${ }^{4}$ Precise probabilistic estimates of existential risks are difficult to produce [20]. Estimates of an existential risk manifesting by the end of the $21^{\text {st }}$ century typically range in the higher single digit or lower double digit percentage probability range, with anthropogenic risks being much more probable and thus much more relevant than natural risks [21, 22.
} 


\section{Prioritization risks}

Prioritization risks arise because the moral goal of colonizing space - creating positive expected future value - can be better achieved by addressing existing risks instead of pursuing space colonization. Delaying space colonization in favor of mitigating existing risks can result in a significantly better long-term future. The specific prioritization risks that I discuss in the subsections below are depicted in Figure 3

Figure 3: Prioritization risks of space colonization.

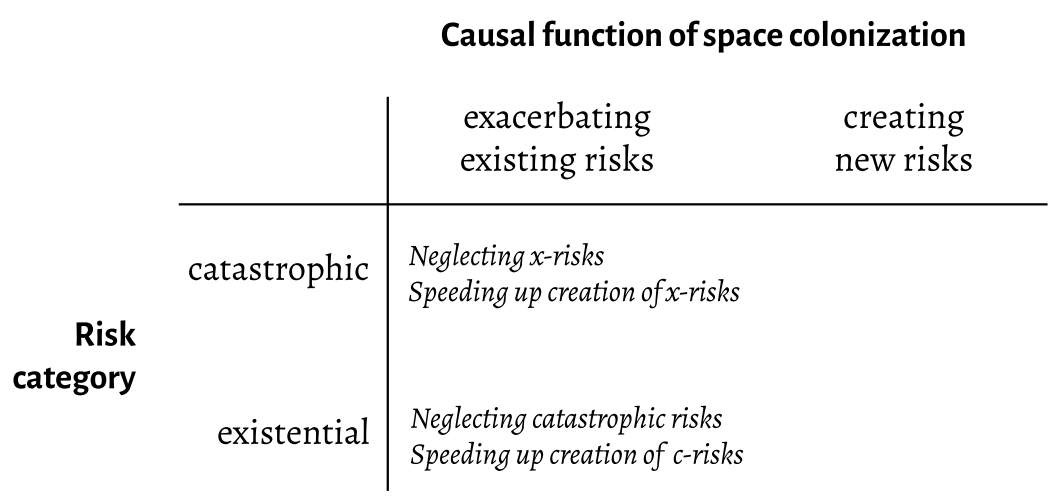

The two prioritization risks I focus on, neglecting existing risks and speeding up the creation of new risks, can be both catastrophic as well as existential in nature.

\subsection{Neglecting existential risks}

Space colonization is, as I argue in the introduction, a generalized strategy for the mitigation of existential risks: If we manage to establish permanent and sustainable habitats beyond Earth, there is a chance that existential risks would either become less probable or cease to be existential at all because not all proverbial eggs are in the same basket. Given this premise, it is tempting to extrapolate it into real-life policy: If space colonization is a hedge against existential risks, then achieving space colonization capabilities must be our top priority.

The problem with this prioritization strategy is that while it might, on its own, increase the probability of space colonization, it ignores the probabilities of existing existential risks. If we want to create as positive a future for humankind (in the sense of increasing humankind's future moral expected value), then we need to weigh the benefits of achieving space colonization 
capabilities sooner rather than later against the benefits of reducing existential risks. When we compare the benefits of these two approaches, as Bostrom [2, 5] argues, it quickly becomes obvious that that the benefits of even marginally reducing existential risks over a given time period are probably much greater than the damage of delaying space colonization by that same time period.

A delay of, say, 100 years in colonizing space has a relatively minor negative impact compared to the immense positive impact of even marginally reducing existential risks in the same time frame. If we, for example, adopt the conservative estimate of $10^{15}$ total future human lives if humankind goes on to enjoy a colonized long-term future [23, then reducing overall existential risks by merely 0.0001 (one percent of one percent) has an expected value of $10^{11}$, or 100 billion, lives. Even the tiniest reduction in existential risks has therefore an immense positive benefit that easily offsets the positive benefit of achieving space colonization capabilities sooner rather than later. This is almost certainly true even if we take into account the potential contribution of space colonization to mitigating existential risks. Early colonization efforts are unlikely to mitigate existential risks as much as targeted existential risk mitigation strategies because the early stages of colonization are bound to be small in scale and fragile. Early habitats would in effect do next to nothing to mitigate existential risks, so creating those early habitats as quickly as possible would do very little in terms of existential risk mitigation.

The practical question that arises with this prioritization risk is how inherently limited resources should be spent. Existential risk mitigation is currently somewhat of an academic niche topic that receives limited public and policy attention, except maybe for the issues climate change mitigation and nuclear safety. Space exploration, on the other hand, is an established policy branch. In 2018 alone, governments spent over 70 billion US dollars on their respective space exploration agencies [24]. Of course, not all of that money is directly used for space colonization effort:5 but space exploration-related public spending is orders of magnitude greater than spending on some important existential risk mitigation areas. For example, the area of AI safety which is concerned with the existantial risk posed by artificial intelligence and the prospect of uncontrollable superintelligence is funded only to the tune of around ten million dollars a year, even though it is currently one of the greates existential risks [25].

\footnotetext{
${ }^{5}$ Arguably, however, all money spent on space exploration ultimately is ultimately spent on space colonization because any advances in understanding and exploring space automatically contribute to our space colonization prospects.
} 


\subsection{Speeding up the rate of existential risk creation}

Achieving space colonization capabilities means obtaining sufficiently advanced technology for venturing beyond Earth and permanently sustaining human life there. In order to achieve that goal, maximizing the pace of technological development seems like an instrumentally desirable goal: The faster we technologically innovate and develop, the higher the probability of obtaining space colonization captabilities. However, the problem with such a technological push is that the increased pace of technological development might also result in an increased pace of existential risk creation.

As I argue above in subsection 2.1, anthropogenic existential risks correlate with human technological development. All non-natural existential risks are the result of our technological progress, and more technological progress is likely to beget more existential risks. Of course, this should not be misunderstood as a quasi-Luddite argument against all technological progress in general. Scientific and technological progress has made life enormously better and removed tremendous amounts of suffering from the world, to the benefit of current as well as all future generations. However, existential risks are an unprecedented challenge, and the more numerous and probable they are, the more difficult it is to mitigate them in time. In the context of space colonization, this issue is of elevated importance because, as I argue above in subsection 3.1, delaying space colonization has an almost imperceptible impact on the long-term future of humankind, whereas existential risks and our attempts at mitigating them (or failing to do so) has an enormous impact.

\subsection{Neglecting acute catastrophic risks}

Humankind is today not only faced with existential, but also with catastrophic risks. The potential damage of catastrophic risks is smaller than that of existential risks, but still not morally negligible. Issues such as (extreme) poverty, the global burden of disease, global animal welfare in farming, and so forth are pressing issues, and they are, in a sense, more acute than existential risks because they are causing enormous moral disvalue right now. The shadow that acute catastrophic risks are casting into the future is not as long as that of existential risks in terms of overall expected disvalue, but in principle, a similar neglect argument as with existential applies in the context of space colonization. Delaying space colonization in favor of mitigating catastrophic risks could in some scenarios yield greater overall moral benefits than achieving space colonization sooner without having mitigated those catastrophic risks.

Imagine, for example, a timeline A in which humankind delays space colo- 
nization in favor of pursuing a cure against all forms of cancer, and a timeline $\mathrm{B}$ in which humankind stresses colonizing space and does not focus on cancer cures. In timeline A, a universal cure is found, and billions of people immediately benefit (they are spared terrible suffering). In addition, all future generations benefit as well. In timeline B, there is no cure, and humankind has started expanding beyond Earth. During the colonization delay in timeline A, billions of people lead much better lives and once humans in timeline A start colonizing space, there is no cancer and the disvalue it creates. In timeline B, however, there would potentially be a colonization-induced population explosion before a cancer cure is eventually developed, which would mean that in timeline $\mathrm{B}$, there is enormous disvalue (the colonizing human population was suffering cancer for a while) that is not offset by the relatively small gain in moral value of achieving space colonization sooner.

The case in favor of prioritizing catastrophic risk mitigation and thus accepting delays space colonization is not as strong as in the context of existential risks. The case for catastrophic risk prioritization is contingent on the size of the catastrophic risk in question. It is possible that not all catastrophic risks are grave enough that they warrant delaying space colonization. In general, the argument against neglecting catastrophic risks should not be misunderstood as the cliché argument of "there are more important problems today than space". The point is explicitly not that mitigating all existing risks should be prioritized over colonizing space, but that some catastrophic risks could create enough future disvalue that delaying space colonization and mitigating them yields the better overall moral outcome.

\subsection{Speeding up the rate of catastrophic risk creation}

Similarly to the risk of speeding up the rate of existential risk creation described in subsection 3.2, an increased pace of technological development might result in an increased pace of catastrophic risk creation. This is unsurprising insofar as existential risks can correlate with existential ones. For example, the artificial intelligence family of technologies has created the existential risk of uncontrollable superintelligence, but it has also created catastrophic risks such as unprecedented cybersecurity risks [26] or the prospect of autonomous weapons systems [27]. An increased pace of catastrophic risk creation is a concern for the same reason as an increased pace in existential risk creation is: The more such risks exist, the more difficult it might be for humankind to adequately mitigate them in time. 


\section{Aberration risks}

Aberration risks are risks inadvertently created by space colonization. They are entirely new risks without precedents in human history, and they expand the moral circle of concern (far) beyond humankind. The disvalue that they can create is potentially orders of magnitude greater than the positive value of humankind's future. Figure 4 is a summary of the specific aberration risks that I focus on.

Figure 4: Aberration risks of space colonization.

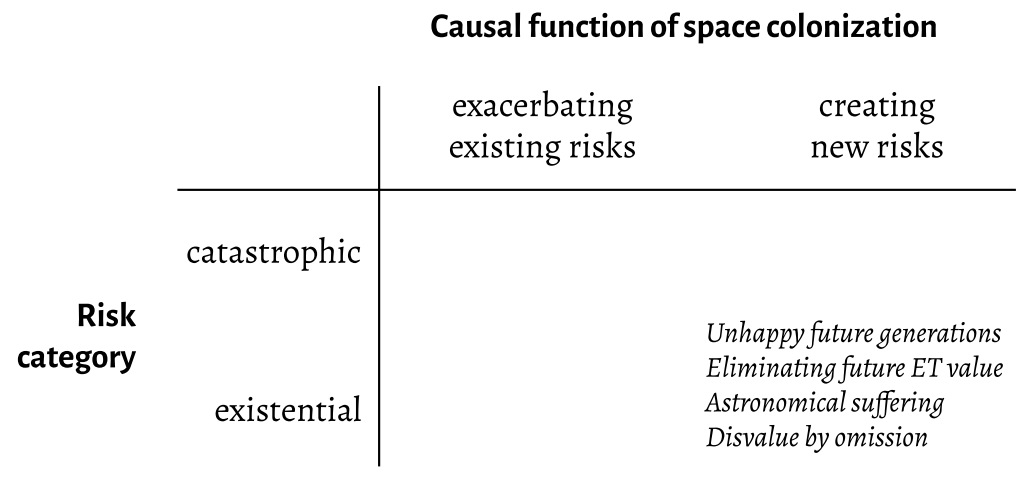

I discuss four specific aberration risks in the subsections that follow; all four of them are existential in nature.

\subsection{Unhappy future generations}

The moral impetus of colonizing space is to increase the probability that all the potential future humans that could live do come into existence. But we do not only want future generations to exist but to live lives that are actually worth living. However, the moral landscape of the far future might turn out to be much blurrier.

Imagine, for example, a scenario in which 10 billion people live on Earth, and another 2 billion people live on a terraformed Venus. The people on Earth are living roughly as good lives as we are today, but the Venusians are all categorically suffering: Because the terraforming was not entirely successful, some of the original toxic Venusian atmosphere remained, leading to mild respiratory issues in most of the population. Is this state of affairs preferable to there not being any Venusians at all? On the face of it, yes. After all, the total total wellbeing of humankind is much greater with the 2 billion Venusians than without them. Mild respiratory issues in 2 billion 
people are a nontrivial amount of disvalue, but their existence still seems preferable to their non-existence.

Let us imagine a second scenario. There are 10 billion people on Earh living lives that are roughly as good as the lives we are currently living, there are 2 billion people on Venus with chronic respiratory issues, and there are 500 million people on Mars. Unfortunately, given Mars' inexistent atmosphere and magnetosphere, cosmic radiation is battering the surface, including human habitats. This has lead to permanent damage in the Martians' DNA, resulting in chronic muscular and skeletal disease. As a result, the whole Martian population is collectively suffering from a hereditary and incurable congenital disease. Is this scenario preferable to there being no Mars colony or to there being no colonization at all? On the face of it, the answer is again yes: Even though the lives of the Martians are much less pleasant than the lives of the Earthlings and the Venusians, the Martians are still living lives that are worth living to them. The total amount of wellbeing and happiness of humankind is greater than if there were no Martians at all.

In these hypothetical scenarios, we are confronted with two classic problems from population ethics: The so-called repugnant conclusion [28] and the non-identity problem [29]. The repugnant conclusion is the observation that our intuitive judgement of moral desirability, the increase of total happiness or welfare, is flawed. In our second scenario, the Martians are living fairly terrible lives that might barely be worth living because they are full of suffering. Just because the total amount of happiness or welfare is greater with the Martians than without them does not mean that a world with the chronically suffering Martians is morally desirable. The repugnant conclusion is relevant in the context of space colonization both because of its existential scope as well as its empirical plausiblity. Creating habitats that are able to permanently sustain human life is an immense technological challenge, and it is not unrealistic to expect that life beyond Earth will be miserable for quite some time.

The non-identity problem is the observation that something is wrong with another common moral intuition. Our Martians live miserable lives, but from their own subjective point of view, that reality is preferable to the alternative of not existing at all. In a sense, no matter how terrible life for the Martians is, no moral harm seems to be done because no person was actively harmed by there being Martians - the Martians are not suffering because someone actively and malevolently hurt them or made them sick; they simply come into existence in their frail, sickly state. This means that if a Martian could choose between existing the way she does and not existing at all, she would almost certainly pick the former. But something is wrong with this conclusion. The Martians are living lives full of suffering, and clearly, this 
state of affairs is morally undesirable.

The concrete problems within scenarios in which the repugnant conclusion and the non-identity problem apply can be described in several ways. For example, if we put moral emphasis on average wellbeing rather than just total wellbeing, we see that the growth of total wellbeing can go hand in hand with a decrease of average wellbeing. This would indicate that something has gone wrong. However, average wellbeing alone might not be a good enough indicator. For example, in an Omelas-like configuration [30], it is conceivable that average wellbeing would increase while a small subset of people endure hellish suffering. That is why another approach to understand these problems of population ethics is to not only focus on happiness and wellbeing, but also on the negative side of the utilitarian coin, suffering: If some situation or decision produces a disproportionate amount of suffering compared to wellbeing, that situation is undesirable

The repugnant conclusion and the non-identity problem are examples of how many billions of future humans could live considerably worse lives than we do today. That would constitute a moral failure on an existential or nearexistential level — humankind would still exist, but the primary result of our expansion beyond Earth would be a gradual erosion of happiness and a gradual accumulation of suffering.

\subsection{Eliminating future extraterrestrial value}

The discussion so far has mostly centered around the moral value of humankind. But in the context of space colonization, the moral reference group is not just humankind. Given the vastness of our galaxy alone, let alone the entire observable universe, the risks of space colonization for beings other than humankind need to be also taken into consideration. This starts with microbial life: Endangering primitive extraterrestrial life through space colonization could destroy immense future moral value.

We do not currently know whether life exists (or has ever existed) beyond Earth. But there is some plausibility to the assumption that the development of life is not a once-in-a-universe event. The conditions that presumably gave rise to life on Earth are almost certainly abundant throughout our galaxy, which means that, statistically speaking, primitive microbial life could come into existence relatively often [32]. If humans engage in space colonization, and if humans come into contact with extraterrestrial life, the extraterrestrial

\footnotetext{
${ }^{6}$ This is a superficial dsecription of negative utilitarianism, or suffering-focues ethics. The question of what kind of suffering should be minimized in which ways is complex. For a comprehensive discussion of that philosophical approach, cf. 31.
} 
life in question will most likely be microbial in nature. What moral obligations do future human colonizers have towards microbial extraterrestrial life? To make this question more concrete, imagine a colonization scenario in the near future: Humans decide to terraform Mars in order to make it habitable for humans, but doing so would kill all existing species of Martian microbes that were discovered not long before the decision to terraform Mars. Would terraforming Mars be morally acceptable?

Microbial life on Earth is non-sentient, and the microbial life on Mars would also, in all likelihood, be non-sentient. If the Martian microbes neither feel anything like happiness nor experience anything like suffering, there are no utilitarian considerations of wellbeing or happiness to be made humans could neither affect their level of wellbeing nor could they rob them of their capacity for happiness since microbial life forms lack both. However, there is a counterargument to this position: The Martian microbes have the potential to evolve into more complex, sentient and possibly even intelligent life forms. Eradicating them would therefore represent an existential damage, because all the potential future moral value would be lost. If this argument seems abstract, consider the scenario if the microbial life in question was Earth-based: If some extraterrestrial intelligence had eradicated our primitive microbial ancestors, humankind (as well as all other sentient Earth-based life) would never have come to be.

A second moral argument in favor of preserving the Martian microbes in our scenario is the argument of intrinsic value 33. According to this position, the moral obligation towards extraterrestrial microbial life is not contingent on its sentience, but on its mere existence: Life in and of itself has a moral value, and by virtue of existing, our Martian microbes have a kind of right to their existence. In addition, and perhaps crucially, we have an obligation to respect that right. This deontological, Kantian view is not concerned with wellbeing and suffering, but instead with rights of and duties towards life. I find the utilitarian view of potential future moral value more useful than the intrinsic value argument, but it is worth mentioning the latter for the sake of completenes.

In any case, both moral arguments, the utilitarian view of potential future moral value as well as the deontological intrinsic value argument, suggest that endangering microbial life could be devastatingly wrong. A logical consequence of such considerations would be to adopt a strongly conservationist stance whereby humans refrain from colonizing a potentially large number of viable celestial bodies lest they threaten the microbes that have evolved there [34]. Such an approach could limit human expansion to entirely artificial habitats and to biologically completely barren moons and planets. 


\subsection{Astronomical suffering}

Space colonization means that humans and human actions will spread beyond Earth and possibly cover, relatively speaking, vast areas of the reachable universe. This will potentially create immense positive value, but it also makes possible a form of existential risks that are astronomical in scope and hellish in severity - that are, in other words, orders of magnitude worse than anything humankind has caused or encountered so far. This subset of extreme existential risks is referred to as suffering risks [35].

Suffering risks are risks that are far worse than humankind going extinct or entering permanent moral stagnation because they mean that the suffering that is created through these risks is far greater than all suffering that has existed on Earth so far. There are different vectors of potential astronomical suffering. For example, it is conceivable that future human generations will spread wildlife throughout the colonized space, either inadvertently or actively. Wild animals on Earth generally lead short, miserable lives full of sometimes the most brutal suffering [36]. In in the history of Earth, wildlife suffering has not really improved at all, so astronomical wildlife suffering would likely represent a constant source of disvalue.

Another vector for suffering risks are sentient simulations. Given growing computational power, it is conceivable that we will eventually be able to simulate sentience, and as soon as simulated sentience is possible, simulated suffering will be as well. This technological path is not necessarily dependent on space colonization, but a colonizing humankind might have greater capabilities for running such simulations, for example by tapping into the power of stars in different Solar systems. Instances of simulated suffering could create more suffering than has ever occurred in the biological universe, within fractions of a second.

The risk of astronomical suffering is more uncertain than other existential risks, but it is at the same time more severe. At stake is not just humankind's total potential positive future moral value, but disvalue that is decoupled from humankind and is potentially many orders of magnitude greater than all the happiness and wellbeing that could be created by human colonization of space.

\subsection{Creating disvalue by omission}

The aberration risks I have outline in the previous subsections are all related to active decisions and actions of humankind. But not doing something can also represent an existential risk in some space colonization scenarios. I call this type of risk creating disvalue by omission. 
One way in which disvalue is created by omission and inaction is related to extraterrestrial sentience and suffering. Given our knowledge of how sentient beings suffer on Earth, it seems plausible to assume that sentient beings beyond Earth also experience suffering. Given this knowledge and given our expected technological future capabilities, we could opt to reduce suffering of sentient beings throughout the parts of the universe we can reach. Not doing so would mean allowing potentially astronomical amounts of suffering to continue existing. This seems morally wrong, since our general moral intuition is that suffering is undesirable and our individuel preference for being suffering-free ourselves.

Another case in which inaction creates disvalue involves extraterrestrial intelligence. For example, if there are other technologically advanced intelligent civilizations in our galaxy 7 , then they are almost certainly faced with similar existential risks as we are today; this is dictated by the causal relationship of technological progress and the emergence of existential risks from that progress. Given this knowledge, humankind seems to have a moral obligation to seek out and help such civilizations, because reducing their existential risks is prima facie at least as morally valuable as reducing our own.

A third cas of creating disvalue by omission involves extraterrestrial intelligence with misaligned morality. It is possible that advanced extraterrestrial civilizations or extraterrestrial artificial intelligences act according to objectively bad moral principles such as sadistic tendencies. Such a civilization or AI could wreak moral havoc on an astronomical scale. Given this possibility, it can be argued that humankind would need to actively seek out extraterrestrial intelligence and "correct" their morality in order to stop the suffering the intelligence in question is causing or could cause. Even if this is only a remote possibility, the potential positive expected value of stopping such activity would be enormous.

The risk of creating disvalue by omission is ultimately a variation of the drowning child problem [38]: If we are cognizant about a moral problem and we have the means for solving that problem (with some degree of confidence), there is no real excuse not to do so.

\footnotetext{
${ }^{7}$ For the sake of brevity, I refrain from a discussion on the plausibility and probability of the existence of intelligent life and technologically advanced civilizations beyond Earth. The Fermi paradox, the observation that our galaxy should be teeming with civilizations but apparently is not, has been discussed for decades, and potential explanations for our apparent solitude are plentiful [37. Suffice to say that it is both plausible and possible that, but very uncertain whether intelligent, technologically advanced extraterrestrial life beyond Earth exists.
} 


\section{Conflict risks}

Conflict risks are risks that are created by the prospect of hostile actors or powers in the context of space colonization. Conflict risks are in principle not unlike conflicts that humankind has experienced throughout its Earth-based history, but they are much greater in scope and severity. The four conflict risks I focus on are depicted in Figure 5.

Figure 5: Conflict risks of space colonization.

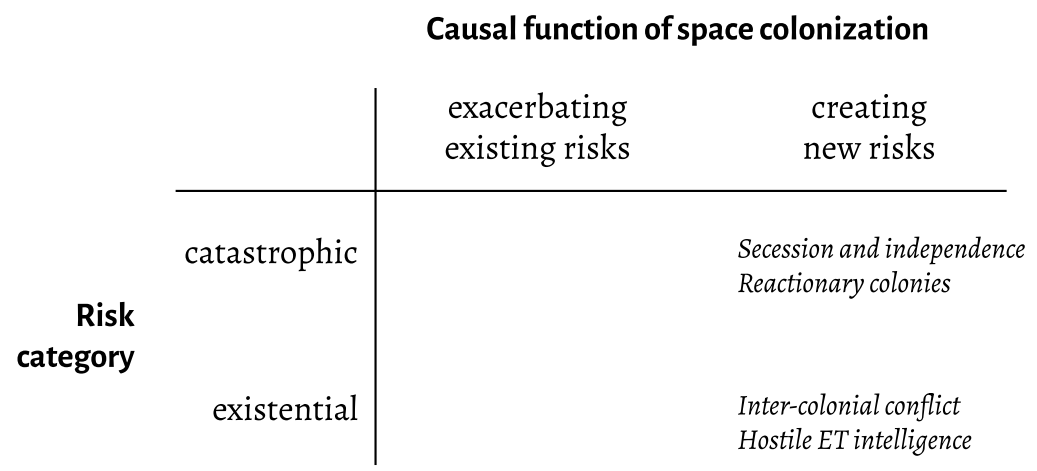

I identify two catastrophic and two existential conflict risks.

\subsection{Secession and independence conflicts}

Human habitats beyond Earth are likely to remain modest in the nearterm future. The International Space Station, humankind's most advanced habitat-like project so far, can accommodate six people and is dependent on supplies from Earth. More ambitious colonization projects such as SpaceX's plan for Mars colonies typically envision what amounts to very small and simple camps [39]. Managing such simple colonization projects should be doable legally and politically. With more mature colonies, however, the picture changes.

Imagine, for example, the large, self-sustaining habitat on Venus that consists of 2 billion people that I mentioned in a thought experiment before. That hypothetical habitat is truly self-sustainable, in the sense that survival on Venus is not contingent in any way on resources or other kinds of support from Earth. If prior human history is an indication, it is conceivable that the Venusians could at some point seek to change their political status. They might want to no longer be governed by Earth or Earth-based governements and instead have sovereignty to autonomously and freely shape Venus' future. 
They might, in other words, seek to seceede and become an independent political entity.

Given prior human history of secession and independence movements, such a claim to independence in the context of space colonization could easily result in violent conflict, and given the scale of the conflict parties in this scenario, the bloodshed could be much greater than all the wars that happened in Earth's history so far. Of course, we do not know what the dominant political philosophy of the future will be. Perhaps popular sovereignty and the wish for autonomy will be fully respected and met with unconditional, enlightened understanding. But that prospect is, at best, uncertain, and the prospect of catastrophic violent conflicts seems at least possible.

\section{$5.2 \quad$ Reactionary colonies}

Let us assume for the sake of argument that the risks surrounding secessionist claims of extraterrestrial colonies will eventually have been overcome and that there are colonies which have attained a country-like or world-like status. What should the political systems in and the moral foundations of those independent colonies look like? Ideally, they would be at least as democratic, liberal, and generally morally progressive as the most democratic, liberal, and morally progressive countries today. More specifically, independent future colonies should have socio-political systems that do not lower average wellbeing or create (disproportionately) more suffering compared to their pre-existing peers such as Earth-based countries (Or whatever the dominant polity on Earth in that future might be.). However, there is no guarantee that independent colonies will meet that socio-political and moral bar. It is possible that there will be colonies whose socio-political systems are regressive in one way or another, marked by a relative moral decay compared to the baseline of political systems and moral frameworks. I call such potential undesirable entities reactionary colonies.

The emergence of reactionary colonies might seem implausible given that humankind has, very roughly speaking, so far morally improved over the course of its history ${ }^{8}$. But reactionary colonies might actually be a fairly common future development. If humankind at some point achieves the technological means for creating colonies with relative ease, creating new colonies might be an attractive option for extremist groups and beliefs. Imagine, for

\footnotetext{
${ }^{8}$ This is not universally true. In many domains, such as medicine, human rights, warfare, and so forth, humankind has arguably made significant moral progress. However, there are also domains in which humankind has starkly deteriorated. For example, many more animals today suffer in intenstive and other forms of farming than did, for example, one thousand years ago.
} 
example, a religious group that believes in the fundamental superiority of men over women. Such a religious group might find it difficult adhering to their flawed moral principles in a pluralistic society. Opting for colonial exodus might represent an attractive opportunity for that religious group to build a society from scratch which is based on their notions of female inferiority and subjugation.

The specific risk posed by reactionary colonies ist twofold. Reactionary colonies would by definition lower the average happiness and wellbeing of humankind and create unnecessary, preventable suffering. Reactionary colonies would also represent potential rogue actors that could greatly amplify the aberration risks described in section 4. For example, a dictatorial regime that causes great suffering to its population might be tempted to expand its dictatorial ideology to other colonies. Or that dictatorial colony could be led by a psychopathic elite that enjoys letting sentient simulations suffer as much as possible. The potential catastrophic and even existential multiplicator effects of reactionary colonies are, unfortunately, numerous.

\subsection{Inter-colonial conflict}

Let us, again for the sake of the argument, assume that the previous problem of reactionary colonies has somehow been solved or avoided. Humankind has continued its path of technological development, and it has established several large clusters of colonies beyond the Solar system. Assuming that the fundamental problem of faster-than-light communication has not been solved yet, communication between the clusters lags months or even years, and physical contact between the clusters is rare since travel takes even longer than communication.

The inevitable consequence of such a splintering of human civilization is that the different clusters of colonies would over time develop distinct cultures, and with only scarce and delayed contact with other clusters, a form of intergroup bias [40], the moral preference of one's own in-group over the out-group, would likely start to manifest. Over time, that us-versusthem heuristic could help create distinct and solidified social identities within the colony clusters [41], and the beliefs and preferences about the outgroup colonies could become more overtly negative. Given enough time and great enough idiosyncratic development within each colony cluster, the cultural and moral connections between the colony clusters could further erode, and in their place, a sense of dread and looming danger about the others' goals and preferences could take hold. Over a long enough period of time and great enough separation, the perception that other colonies are a threat could grow; so much so that taking preventative action and attacking and suppressing 
them might seem like the most rational course of action [42]. Given the scale and the likely technological sophistication of future weapons systems, a violent conflict between advanced colonies and colony clusters would create suffering on an astronomical scale.

Of course, the prospect of inter-colonial conflict is somewhat speculative [43. But given humankind's past experiences, violent conflict clearly seems within the realm of the possible. That does not mean that such an almost immeasurably terrible conflict is unavoidable. Even the slightest probability of such conflict, however, means immense potential expected disvalue.

\subsection{Hostile extraterrestrial intelligence}

We do not currently know whether intelligence, biological or artificial, exists beyond Earth, but it is not implausible to assume so. Coming into contact with as well as refraining from seeking contact with extraterrestrial intelligence poses risks, as I discuss in section 4. In addition to these risks, extraterrestrial intelligence also poses a conflict risk: If an extraterrestrial intelligence has moral values that are sufficiently different form humankind's, the intelligence could de facto react to humankind and other forms of intelligence with hostility.

At first glance, the prospect of a hostile extraterrestrial intelligence, be it biological or artificial, might seem far fetched. Isn't some baseline of altruistic moral behavior to be expected of any kind of intelligence? We might intuitively associate intelligence with prosocial behavior of one kind or the other, but from an evolutionary perspective, greater intelligence is not necessarily correlated with prosocial and altruistic behavior towards other species [44]. Intelligence is the generalized capability for achieving goals, regardless of moral underpinnings and considerations. After all, that is why artificial intelligence as superintelligence is an existential risk today: Superintelligence is a threat precisely because it is much more intelligent than humans but lacks any inherent sense of human preferences and moral values.

Hostile extraterrestrial intelligence is a serious risk even if such intelligence is exceedingly rare in the universe. The mere possibility of hostile intelligence could result in something like a "Dark Forest" [45] game theoretic configuration. If benevolent biological or artificial intelligence is aware of the possibility of hostile alien intelligence, the best course of action is silence (If there might be hostile alien intelligence, best not to be noticed.). In addition, preventative action could also be a rational choice: If an alien intelligence - such as our human civilization - is being observed gradually expanding beyond our home planet, a risk averse intelligence, even one that is benevolent in principle, might find it prudent to eliminate that civilization; 
be it because the civilization in question might become hostile or because it might, through folly or ineptitude, draw unwanted attention to oneself. The mere possibility of fundamtenally hostile intelligence could, in that sense, be enough to fuel a silent astronomical war.

\section{Discussion}

\subsection{Is space colonization desirable in light of its risks?}

In the previous sections, I have outlined some prioritization, aberration and conflict risks that could create enormous disvalue - potentially many times greater than the total positive future expected value of humankind. In light of such risks, we have to ask whether space colonization is desirable at all. The answer to this question depends on the moral framework we use to answer it.

The moral perspective of the present article is, broadly speaking, utilitarian in scope: Space colonization matters because of humankind's potential positive future value, and the risks of space colonization matter because of their potential negative disvalue. However, deciding what matters how much or even categorically more depends on the flavor of utilitarianism one adheres to. Casper [46], inspired by discussions on the merits of negative utilitarianism [47, 48, proposed a simple one-dimensional scale to distinguish between negative, suffering-focused varieties of utilitarianism and positive, happinessfocused varieties. The scale is is depicted in Figure 6.

Figure 6: Utilitarianism scale by Casper [46].

\begin{tabular}{|c|c|c|c|c|c|}
\hline $\begin{array}{c}\text { strong } \\
\text { negative }\end{array}$ & $\begin{array}{l}\text { threshold } \\
\text { negative }\end{array}$ & $\begin{array}{c}\text { weak } \\
\text { negative }\end{array}$ & $\begin{array}{c}\text { weak } \\
\text { positive }\end{array}$ & $\begin{array}{l}\text { threshold } \\
\text { positive }\end{array}$ & $\begin{array}{l}\text { strong } \\
\text { positive }\end{array}$ \\
\hline
\end{tabular}

Strong negative utilitarianism means that only suffering and reducing suffering matters. Strong positive utilitarianism accordingly means that only happiness and increasing happiness matter. Threshold negative utilitarianism means that there is some threshold of suffering beyond which no amount of happiness can compensate it; threshold positive utilitarianism means the opposite, that there is a threshold of happiness beyond which no amount of suffering can outweigh it. Weak negative utilitarianism means that there is an asymmetry between suffering and happiness; happiness matters, but suffering should receive greater weight. Correspondingly, weak positive utili- 
tarianism sees suffering and happiness as commensurable, but places greater weight on happiness.

I personally tend towards weak negative utilitarianism. Happiness and wellbeing matter, but an equal amount of suffering does not only nullify that happiness but actually create a moral net negative. Indeed, large portions of the argument in this article only make sense if one adopts a negative flavor of utilitarianism (Or possibly one in which happiness and suffering are regarded as perfectly equal in moral weight.). From the point of view of weak negative utilitarianism, space colonization can be regarded as a worthwile effort if and only if the accompanying risks of space colonization that can create equal or greater disvalue are mitigated. The net moral outcome needs to be positive, and very clearly so. From a utilitarian perspective closer to the strong negative interpretation, however, the prospect of space colonization can be regarded as categorically undesirable given the risks; at least as long as there is no way to completely mitigate the most grave ones such as astronomical suffering.

If we adopt a weakly negative utilitarian view of space colonization and its risks, we must emphasize risk management and mitigation. But this forces us to face another question: Is it even possible to meaningfully influence the long-term future?

\subsection{Is the long-term future tractable?}

Humans are notoriously bad at predicting even the short-term future [49]. Given our very limited predictive skill, even thinking of influencing the longterm future might seem dubious? 9 . As we move further into the future, uncertainty about both our knowledge and the impact of today's actions on the future grows [50].

In light of the fundamentally uncertain nature of the long-term future, any long-term oriented action today might seem misplaced. But there are a few reasons to be optimistic about our ability to positively influence the longerterm future ${ }^{10}$. For example, while many of the potential measures for making space colonization safer aim to maximize long-term future moral value, the measures themselves are usually aimed at the short-term and medium-term future. For example, mitigating existential risks over the next 100 years can have tremendous positive impact for the next several thousands of years -

\footnotetext{
${ }^{9}$ As mentioned in the introduction, I define the long-term future on a timescale of thousands of years; the long long-term future of humankind could play out on timescales of millions of years.

${ }^{10}$ The arguments I present here are qualitative and non-technical. For a more systematic discussion of the issue, see 23 .
} 
we do not know what those millennia will look like for humankind, but we do know with certainty that mitigating existential risks today will shape them in a positive way.

Another way in which short- and medium-turn measures could positively influence the uncertain long-term future is through simple agenda setting. The mere fact that we are starting to think about long-term challenges today could put into motion positive dynamics of prioritization for the future. If we start discussing the safety of space colonization today, long before humankind is close to large-scale colonization capabilities, we are increasing the probability that appropriate safety measures will be implemented soon enough so that they can be effective. This is true even if the contribution of today's actions is miniscule.

Furthermore, evidence from human history suggests that historical trajectories are not purely stochastic in nature. For example, the discrepancy in democratic performance between northern and southern Italy can be understood as a demonstration of inertia or parth-dependency of history. The modern institutional makeup of Italy is mostly the same, but the strength of civil society and the performance of the democratic institutions is not. These differences today are at least to some degree caused by different governance systems established in northern and southern Italy about one thousand years earlier [51].

Overall, there is some plausiblity to the belief that we can positively affect the long-term future in general as well as in the context of space colonization. If we want to start mitigating the risks of space colonization today, the best vector for doing so is governance. Unfortunately, that is easier said than done.

\subsection{The challenge of space colonization governance}

The cornerstone of space-related governance is the Outer Space Treaty from 1967 [52]. Given its historical Cold War context, the Outer Space Treaty is a remarkable achievement. The United States and the Soviet Union, among other signatory countries, agreed not to deploy weapons of mass destruction into space, and they declared outer space a common good that cannot be appropriated by any country, organization or individual. Unfortunately, this promising start of space governance was not followed up with additional agreements and treaties that would serve as a binding international framework. There have been some additions over time, such as the Rescue Agreement of 1968, the Space Liability Convention of 1972, and the Registration Convention of 1976 [53], there has been little to no progress for decades. The overall pace of space-related international law-making has slowed down in 
recent decades [54].

The result of this state of affairs is that the existing governance foundation built around the Outer Space Treaty is fraying, if not outright falling apart. The governance solution of the International Space Station, for example, was realized with a custom legal agreement between the participating countries [55]; there was simply no prior governance framework within which the operation of the ISS could have been placed. The relative vacuum surrounding space governance has also lead to unilateral ambitions. For example, the American Space Commerce Free Enterprise Act of 2017 seeks to proclaim the United States' unilateral right to commercial exploitation of outer space and celestial bodies, in direct violation of the Outer Space Treaty (At the time of this article, the bill was still with the US Senate and not yet enacted into law.). In April of 2020, US President Donald Trump signed an executive order declaring the United States' interest in and right to commercial exploitation of the Moon and other celestial bodies [56]. In May of 2020, NASA unilaterally released the so-called Artemis Accords which it hopes will serve as a form of governance foundation for the exploration and commercial exploitation of the moon [57]. However, the Artemis Accords are explicitly not intended as a global multilateral governance framework. They are merely a set of bilateral agreements between the United States and countries which will participate in NASA's Artemis missions [58].

Overall, it seems fair to say that space governance is in shambles today. Creating any kind of meaningful space colonization-related governance in such a policy and policymaking environment is difficult, to say the least. We should not expect governance work on space colonization be initiated by governmental actors any time soon, so the proverbial ball is, at the time being, probably in the academic court. If we were to draft a space colonization governance framework that would be effective at mitigating colonization-related risks and maximize the positive future value, what are some factors or aspects that need to be taken into account?

First, we should consider a break with the past. Existing space governance based on the Outer Space Treaty has barely seen any progress over the decades, and the Outer Space Treaty does not seem geared towards questions of space colonization risks. Starting with a philosophical clean slate that is divorced from the realities of the 1960-ies is probably the easiest way forward.

Second, given the uncertainty of the long-term future, a governance framework for space colonization should be conceptualized as provisional and malleable. Major principles of safe space colonization might very well be universal, but the empirical realities on the ground might change in the not too distant future. This means that, on one hand, our understanding of space colonization-related risks will almost certainly change over time. The 
practical reality of policymaking on Earth, on the other hand, will probably also undergo significant changes in the future. The current political order on Earth has been, roughly speaking, stable since the Second World War, and it seems plausible to expect the global political order to roughly continue along those lines for several more decades. This means that any governance framework that is geared towards today's workings of global policymaking should aim to achieve tangible results as soon as possible, before the world changes so much that the governance framework and its bodies simply become obsolete. The philosophical timescale of such a governance project thousands to millions of years, but the practical timescale for achieving results should be decades.

Third, and perhaps most importantly, any such exercise in governance building can only be effective if it ranks highly enough on the agenda of the public and of policymakers. We therefore need strategies to "jump start" attention to the issue. One possibility to do so would be to build coalitions with prominent space exploration and space colonization advocates, such as, for example, wealthy and influential space entrepreneur: ${ }^{11}$. Such coalitions would perhaps have some morally dubious aspects to them (teaming up with people who do not necessarily have humankind's best interests but rather their own bottom line in mind is questionable), but there is some prior evidence to suggest that that might be an effective path nonetheless. For example, a 2015 open letter urging research into AI safety [59] arguably brought important public and policy attention to the issue of AI safety thanks to the mere fact that many of the signatories were prominent people with significant public presence and influence. Of course, this anecdote is a limited form of evidence, but early, broad and publicly visible coalition-building seems like an important tactic to consider.

\section{Conclusion}

Space colonization, the establishment of permanent human habitats beyond Earth, has an enormous potential moral value because successful space colonization is the necessary condition for trillions of future people to come into existence. At the same time, however, space colonization is not risk-free. The risks of space colonization are so grave that, in a bad-case scenario, successful space colonization could create more moral disvalue than value; possibly even orders of magnitude more.

\footnotetext{
${ }^{11}$ Examples at the time of the writing of this article are Elon Musk, Jeff Bezos or Richard Branson.
} 
In this article, I have outlined three types of risks that are created by space colonization: Prioritization risks, aberration risks, and conflict risks. Prioritization risks are risks whereby existing catastrophic and existential risks are exacerbated because of colonization efforts. Aberration risks are newly created risks due to space colonization which expand the moral circle beyond humankind and which can potentially create disvalue orders of magnitude greater than all of humankind's potential positive future value. Conflict risks are risks related to violent conflicts caused by hostile intelligence, human or otherwise. These risk types and the specific risks within them are not an exhaustive list of all risks that are created by space colonization, but they are illustrative of the fact that the risks of space colonization is a priority that warrants scientific and public attention. In an only slightly poetically exaggerated way, space colonization can be considered the single most important juncture of human history — so much is at stake that all suffering and all happiness that has ever existed on Earth simply pales in comparison.

The issue of space colonization risks should spill over from academia into public life as soon as possible. Academic research and debate is certainly necessary in order to further map out the problem, but ultimately, our collective goal, I believe, is to establish empirically and philosophically sensible real-world governance in order to make space colonization as safe as possible. Reducing the risks of space colonization even by a tiny fraction means an enormous increase in expected moral value, or an even greater decrease in expected moral disvalue.

The future in which humankind colonizes space is vast, and so are both the potential happiness as well as the potential suffering we can fill that future with. 


\section{References}

[1] G. Mace. Getting the measure of extinction. People \& the Planet, 7(4):9, 1998.

[2] Nick Bostrom. Astronomical Waste: The Opportunity Cost of Delayed Technological Development. Utilitas, 15(3):308-314, November 2003.

[3] Nicholas Beckstead. On the overwhelming importance of shaping the far future. PhD thesis, Rutgers University - Graduate School - New Brunswick, 2013.

[4] Seth D. Baum, Stuart Armstrong, Timoteus Ekenstedt, Olle Häggström, Robin Hanson, Karin Kuhlemann, Matthijs M. Maas, James D. Miller, Markus Salmela, Anders Sandberg, Kaj Sotala, Phil Torres, Alexey Turchin, and Roman V. Yampolskiy. Long-term trajectories of human civilization. Foresight, 21(1):53-83, March 2019.

[5] Nick Bostrom. Existential Risk Prevention as Global Priority. Global Policy, 4(1):15-31, February 2013.

[6] Phil Torres. Existential risks: a philosophical analysis. Inquiry, 0(0):126, August 2019.

[7] Steven Pinker. Enlightenment Now: The Case for Reason, Science, Humanism, and Progress. Penguin, February 2018.

[8] Thomas Moynihan. Existential risk and human extinction: An intellectual history. Futures, 116:102495, February 2020.

[9] Toby Ord, Rafaela Hillerbrand, and Anders Sandberg. Probing the improbable: methodological challenges for risks with low probabilities and high stakes. Journal of Risk Research, 13(2):191-205, March 2010. Publisher: Routledge _eprint: https://doi.org/10.1080/13669870903126267.

[10] Milan M. Ćirković, Anders Sandberg, and Nick Bostrom. Anthropic Shadow: Observation Selection Effects and Human Extinction Risks. Risk Analysis, 30(10):1495-1506, 2010. _eprint: https://onlinelibrary.wiley.com/doi/pdf/10.1111/j.15396924.2010.01460.x.

[11] Stephen J. Brown, William Goetzmann, Roger G. Ibbotson, and Stephen A. Ross. Survivorship Bias in Performance Studies. The Review of Financial Studies, 5(4):553-580, October 1992. Publisher: Oxford Academic. 
[12] John Leslie. The doomsday argument. The Mathematical Intelligencer, 14(2):48-51, March 1992.

[13] Nick Bostrom. Anthropic Bias: Observation Selection Effects in Science and Philosophy. Routledge, October 2013.

[14] John G. Sotos. Biotechnology and the lifetime of technical civilizations. International Journal of Astrobiology, 18(05):445-454, October 2019. arXiv: 1709.01149.

[15] Nick Bostrom. Superintelligence: Paths, Dangers, Strategies. Oxford University Press, Oxford, United Kingdom ; New York, NY, reprint edition edition, May 2016.

[16] Stuart Russell. Human Compatible: Artificial Intelligence and the Problem of Control. Viking, New York?, 2019.

[17] Steven C. Sherwood and Matthew Huber. An adaptability limit to climate change due to heat stress. Proceedings of the National Academy of Sciences, 107(21):9552-9555, May 2010.

[18] Peter Kareiva and Valerie Carranza. Existential risk due to ecosystem collapse: Nature strikes back. Futures, 102:39-50, September 2018.

[19] Piers Millett and Andrew Snyder-Beattie. Existential Risk and CostEffective Biosecurity. Health Security, 15(4):373-383, August 2017. Publisher: Mary Ann Liebert, Inc., publishers.

[20] Simon Beard, Thomas Rowe, and James Fox. An analysis and evaluation of methods currently used to quantify the likelihood of existential hazards. Futures, 115:102469, January 2020.

[21] Thomas Rowe and Simon Beard. Probabilities, Methodologies and the Evidence Base in Existential Risk Assessments. Working Paper, Centre for the Study of Existential Risk, Cambridge, UK, 2018.

[22] Toby Ord. The Precipice: Existential Risk and the Future of Humanity. Hachette Books, New York, March 2020.

[23] Hilary Greaves and William MacAskill. The case for strong longtermism. Technical Report 7-2019, Global Priorities Institute, Oxford, UK, 2019.

[24] Government Space Programs: Benchmarks, Profiles \& Forecasts to 2028. Technical report, Euroconsult, Paris, France, 2019. 
[25] John Halstead. Existential Risk Cause Area Report. Technical report, Founders Pledge, London, 2019.

[26] Miles Brundage, Shahar Avin, Jack Clark, Helen Toner, Peter Eckersley, Ben Garfinkel, Allan Dafoe, Paul Scharre, Thomas Zeitzoff, Bobby Filar, Hyrum Anderson, Heather Roff, Gregory C. Allen, Jacob Steinhardt, Carrick Flynn, Seán Ó hÉigeartaigh, Simon Beard, Haydn Belfield, Sebastian Farquhar, Clare Lyle, Rebecca Crootof, Owain Evans, Michael Page, Joanna Bryson, Roman Yampolskiy, and Dario Amodei. The Malicious Use of Artificial Intelligence: Forecasting, Prevention, and Mitigation. arXiv:1802.07228 [cs], February 2018. arXiv: 1802.07228.

[27] Mark Gubrud. Stopping killer robots. Bulletin of the Atomic Scientists, 70(1):32-42, January 2014. Publisher: SAGE Publications.

[28] Derek Parfit. Overpopulation and the Quality of Life. In Torbjörn Tännsjö and Jesper Ryberg, editors, The Repugnant Conclusion, volume 15, pages 7-22. Springer Netherlands, Dordrecht, 2004.

[29] Derek Parfit. Future People, the Non-Identity Problem, and PersonAffecting Principles. Philosophy \&f Public Affairs, 45(2):118-157, 2017.

[30] Ursula K. Le Guin. The Ones Who Walk Away from Omelas (Variations on a theme by William James). Utopian Studies, 2(1/2):1-5, 1991. Publisher: Penn State University Press.

[31] Magnus Vinding. Suffering-Focused Ethics: Defense and Implications. Independently published, May 2020.

[32] Christopher F. Chyba and Kevin P. Hand. Astrobiology: The Study of the Living Universe. Annual Review of Astronomy and Astrophysics, 43(1):31-74, 2005.

[33] Charles Cockrell and Center for Environmental Philosophy, The University of North Texas. The Value of Microorganisms. Environmental Ethics, 27(4):375-390, 2005.

[34] Kelly C. Smith. The Curious Case of the Martian Microbes: Mariomania, Intrinsic Value and the Prime Directive. In The Ethics of Space Exploration, Space and Society, pages 195-208. Springer, Cham, 2016.

[35] Brian Tomasik. Risks of Astronomical Future Suffering, April 2015.

[36] Brian Tomasik. The Importance of Wild-Animal Suffering. Relations: Beyond Anthropocentrism, 3:133, 2015. 
[37] Stephen Webb. If the Universe Is Teeming with Aliens ... WHERE IS EVERYBODY?: Fifty Solutions to the Fermi Paradox and the Problem of Extraterrestrial Life. Springer Science \& Business Media, October 2002 .

[38] Peter Singer. Famine, Affluence, and Morality. Philosophy \& Public Affairs, 1(3):229-243, 1972.

[39] Elon Musk. Making Humans a Multi-Planetary Species. New Space, 5(2):46-61, June 2017.

[40] Miles Hewstone, Mark Rubin, and Hazel Willis. Intergroup Bias. Annual Review of Psychology, 53(1):575-604, 2002.

[41] Miles Hewstone and Katy Greenland. Intergroup Conflict. International Journal of Psychology, 35(2):136-144, 2000.

[42] Phil Torres. Space colonization and suffering risks: Reassessing the "maxipok rule". Futures, 100:74-85, June 2018.

[43] Milan M. Ćirković. Space colonization remains the only long-term option for humanity: A reply to Torres. Futures, 105:166-173, January 2019.

[44] Douglas Raybeck. Predator-Prey Models and Contact Considerations. In Douglas A. Vakoch, editor, Extraterrestrial Altruism: Evolution and Ethics in the Cosmos, The Frontiers Collection, pages 49-63. Springer, Berlin, Heidelberg, 2014.

[45] Chao Yu. The Dark Forest Rule: One Solution to the Fermi Paradox. Journal of the British Interplanetary Society, 68:142-144, 2015.

[46] Stephen Casper. A Response to Negative Utilitarianism, July 2019. Library Catalog: medium.com.

[47] Toby Ord. Why I'm Not a Negative Utilitarian, 2013.

[48] Brian Tomasik. Three Types of Negative Utilitarianism, 2019.

[49] Philip E. Tetlock. Expert political judgment: How good is it? How can we know? Princeton University Press, Princeton Oxford, new edition edition, 2017. OCLC: on1007271799.

[50] Christian Tarsney. The Epistemic Challenge to Longtermism. Working Paper, Global Priorities Institute, Oxford, 2019. 
[51] Robert D. Putnam, Robert Leonardi, and Raffaella Y. Nanetti. Making Democracy Work: Civic Traditions in Modern Italy. Princeton University Press, Princeton, NJ, 1 edition edition, May 1994.

[52] Catharine A. Conley. Outer Space Treaty. In Encyclopedia of Astrobiology, pages 1-1. Springer, Berlin, Heidelberg, 2014.

[53] Francis Lyall and Paul B. Larsen. Space Law: A Treatise 2nd Edition. Routledge, Abingdon, Oxon ; New York, NY, 2 edition edition, December 2017.

[54] Gennady M. Danilenko. International law-making for outer space. Space Policy, 37:179-183, August 2016.

[55] Lawrence J. DeLucas. International space station. Acta Astronautica, 38(4):613-619, February 1996.

[56] Donald J. Trump. Executive Order on Encouraging International Support for the Recovery and Use of Space Resources, 2020. Library Catalog: www.whitehouse.gov.

[57] Leah Crane. Lunar laws of the land. New Scientist, 246(3284):14, May 2020 .

[58] NASA: Artemis Accords, 2020. Library Catalog: www.nasa.gov.

[59] Stuart Russell, Daniel Dewey, and Max Tegmark. Research Priorities for Robust and Beneficial Artificial Intelligence. AI Magazine, 36(4):105114, December 2015. Number: 4. 